\title{
The comparison of clinical and biological characteristics between IDH1 and IDH2 mutations in gliomas
}

Hao-Yuan Wang ${ }^{1,3,5+}$, Kai Tang ${ }^{2,5+}$, Ting-Yu Liang ${ }^{4,5+}$, Wei-Zhong Zhang ${ }^{1,3}$, Ji-Ye Li $\mathrm{L}^{4,6}$, Wen Wang ${ }^{2,5}$, Hui-Min Hu ${ }^{4,5}$, Ming-Yang Li $i^{4,5}$, Hui-Qing Wang ${ }^{1,3}$, Xiao-Zheng He ${ }^{1,3}$, Zhi-Yuan Zhu ${ }^{1,3}$, Yan-Wei Li ${ }^{2,4,5^{*}}$ and Shi-Zhong Zhang ${ }^{1,3^{*}}$

\begin{abstract}
Background: Mutations in isocitrate dehydrogenase 1 (IDH1) and isocitrate dehydrogenase 2 (IDH2) are frequent in low-grade gliomas and secondary glioblastomas (sGBM). Because they yield the same oncometabolite, D-2-hydroxyglutarate, they are often treated as equivalent and pooled. The objective of this study was to provide insight into the differences between IDH1 and IDH2 mutant gliomas.

Methods: To investigate the different clinical and molecular characterization between IDH1 mutant and IDH2 mutant gliomas, we studied 811 patients with IDH1 mutations, IDH2 mutations and IDH1/2 wild-type. In addition, whole-transcriptome sequencing and DNA methylation data were used to assess the distribution of genetic changes in IDH1 and IDH2 mutant gliomas in a Chinese population-based cohort.

Results: Among 811 gliomas in our cohort, 448 cases (55.2\%) harbored an IDH1 mutation, 18 cases (2.2\%) harbored an IDH2 mutation and 345 cases (42.6\%) harbored an IDH1/2 wild-type. We found that IDH1 and IDH2 are mutually exclusive in gliomas, and IDH2 mutations are mutually exclusive with PTEN, P53 and ATRX mutations. Patients with IDH2 mutations had a higher frequency of $1 p / 19 q$ co-deletion $(p<0.05)$ than IDH1 mutant patients. In addition, a Gene Set Enrichment Analysis (GSEA) showed that IDH2 mutant gliomas were associated with the oxidative phosphorylation gene set, and the four most representative biological processes for genes commonly altered by hypermethylation in IDH2 mutant gliomas were the regulation of cell proliferation, cell motion, cell migration and response to hypoxia. Patients with IDH2 mutant gliomas exhibited longer Overall survival $(\mathrm{OS})(p<0.05)$ and longer Progression-free survival (PFS) $(p<0.05)$ than patients with $1 D H 1 / 2$ wild-type gliomas. However, their OS and PFS did not differ from that of IDH1 mutant patients.

Conclusions: Our study revealed an intrinsic distinction between IDH1 and IDH2 mutant gliomas, and these mutations should be considered separately because their differences could have implications for the diagnosis and treatment of IDH1/2 mutant gliomas.
\end{abstract}

Keywords: Glioma, IDH mutation, Whole transcriptome sequencing, DNA methylation analyzes

\footnotetext{
* Correspondence: liuyanwei_tiantan@163.com; shizhong_zh@163.com

${ }^{\dagger}$ Equal contributors

${ }^{2}$ Department of Neurosurgery, Beijing Tiantan Hospital, Capital Medical University, No. 6 TiantanXili, Dongcheng District, Beijing 100050, China

'Department of Neurosurgery, Zhujiang Hospital, Southern Medical

University, 253\# Gongye Road, Guangzhou, China

Full list of author information is available at the end of the article
} 


\section{Background}

Isocitrate dehydrogenase $(I D H)$ enzymes encode the $\mathrm{NADP}^{+}$-dependent isocitrate dehydrogenase, which catalyzes the oxidative decarboxylation of isocitrate to form an $\alpha$-ketoglutarate $(\alpha-\mathrm{KG})$. IDH1 and IDH2 proteins share a high degree of sequence similarity $(70 \%$ in humans) and are encoded by distinct genes (IDH1, 2q33 and $I D H 2,15 q 26)$. Mutations in IDH1 and $I D H 2$, which represent the most frequently mutated metabolic genes in human cancer, are implicated to be mutated in more than $50-80 \%$ of low-grade gliomas and secondary glioblastomas (sGBM), $10 \%$ of intrahepatic cholangiocarcinoma, $20 \%$ of acute myeloid leukemia (AML), $56 \%$ of chondrosarcomas, and over $10 \%$ of melanoma cases [1-5]. Although $I D H 1$ and $I D H 2$ are highly similar and catalyze identical reactions, $I D H 1$ is localized in the cytosol and $I D H 2$ is found in the mitochondrial matrix. In addition, the spectrum of cancers and their subtypes are different. For example, IDH1 mutations are predominant in gliomas, chondrosarcoma, and cholangiocarcinoma, whereas IDH1 mutations and IDH2 mutations are equally common in AML. Despite their different physiological characteristics, most genomic studies of the molecular landscapes in human cancer have frequently combined IDH1 mutations and IDH2 mutations as a single functional group.

Glioma, the most common primary brain tumor, is classified as grade I to IV based on histopathological and clinical criteria established by the 2007 World Health Organization (WHO) [6]. WHO grade I gliomas are often curable by surgical resection, whereas WHO grade II or III gliomas are invasive and have a poor prognosis. WHO grade IV tumors (glioblastomas), the most invasive tumors, feature a median survival of only 16 months, even after aggressive treatment consisting of surgery, radiation therapy, and chemotherapy [7]. In 2008, the genes encoding $I D H 1$ were found to be mutated in low-grade gliomas and a subset of sGBM [8]. In subsequent studies, IDH1 mutations were reported to occur in $70-80 \%$ of WHO grade II or III astrocytomas, oligodendrogliomas, and oligoastrocytomas, whereas a small group (3-5 \%) were found to harbor IDH2 mutations [1]. This pattern contrasts that observed in AML, which features similar rates of IDH1 (6.6 \%) and IDH2 mutations (10.8\%) [9]. Moreover, mutations of $I D H 1$ and $I D H 2$ are mutually exclusive in gliomas, and biochemical investigations showed that $I D H 1$ and IDH2 mutations differ in D-2-hydroxyglutarate (D-2HG) production in gliomas [10]. This difference suggests that $I D H 1$ and $I D H 2$ mutations may impact different cellular pathways and exert different tumorigenic effects. To investigate the different clinical and molecular characterization between IDH1 mutant and IDH2 mutant gliomas, we studied a cohort of 811 patients consisting 448 IDH1 mutant, 18 IDH2 mutant and 345 IDH1/2 wild-type gliomas. We performed whole-transcriptome sequencing and DNA methylation analyses of the samples obtained from patients. We compared the mutational landscapes of $I D H 1$ and $I D H 2$ mutant gliomas, their clinical associations, overall survival, and progression-free survival. Our aim was to provide insight into the differences between $I D H 1$ and IDH2 mutant gliomas.

\section{Methods}

\section{Patients and tumor samples}

Glioma samples were obtained from 811 patients with gliomas, including $448 \mathrm{IDH} 1$ mutant, $18 \mathrm{IDH} 2$ mutant and 345 IDH1/2 wild-type gliomas, which were composed of 577 low grade (II + III) gliomas, including 193 diffuse astrocytoma, 39 anaplastic astrocytomas, 49 low-grade oligodendrogliomas, 27 anaplastic oligodendrogliomas, 186 oligoastrocytomas, 83 anaplastic oligoastrocyotmas and 234 glioblastomas. These patients underwent surgery and were followed-up at Beijing Tiantan hosipital from 2004 to 2014.

Table 1 The summary of the materials analysed in this study

\begin{tabular}{llllll}
\hline Pathological diagnosis & WHO grade & $n=811$ & IDH1 mutation No. (\%) & IDH2 mutation No. (\%) & IDH mutation total No. (\%) \\
\hline Diffuse astrocytoma & II & 193 & $138(71.5)$ & $2(1.0)$ & $140(72.5)$ \\
Anaplastic astrocytoma & III & 39 & $14(35.9)$ & $0(0)$ & $3(45.9)$ \\
Oligodendroglioma & II & 49 & $38(77.6)$ & $19.1)$ & $41(83.7)$ \\
Anaplastic oligodendroglioma & III & 27 & $19(70.4)$ & $9(3.7)$ & $20(74.1)$ \\
Oligoastrocytoma & II & 186 & $147(79.0)$ & $1(1.2)$ & $156(83.8)$ \\
Anaplastic oligoastrocytoma & III & 83 & $47(56.6)$ & $16(2.8)$ & $48(57.8)$ \\
Subtotal (grades II and III) & & 577 & $403(69.8)$ & $1(0.5)$ & $419(72.6)$ \\
Primary GBM & IV & 205 & $29(14.1)$ & $1(3.4)$ & $30(14.6)$ \\
Secondary GBM & IV & 29 & $16(55.2)$ & $2(0.9)$ & $17(58.6)$ \\
Subtotal (Glioblastoma) & IV & 234 & $45(19.2)$ & $18(2.2)$ & $47(20.1)$ \\
Total & & 811 & $448(55.2)$ & $466(57.4)$
\end{tabular}




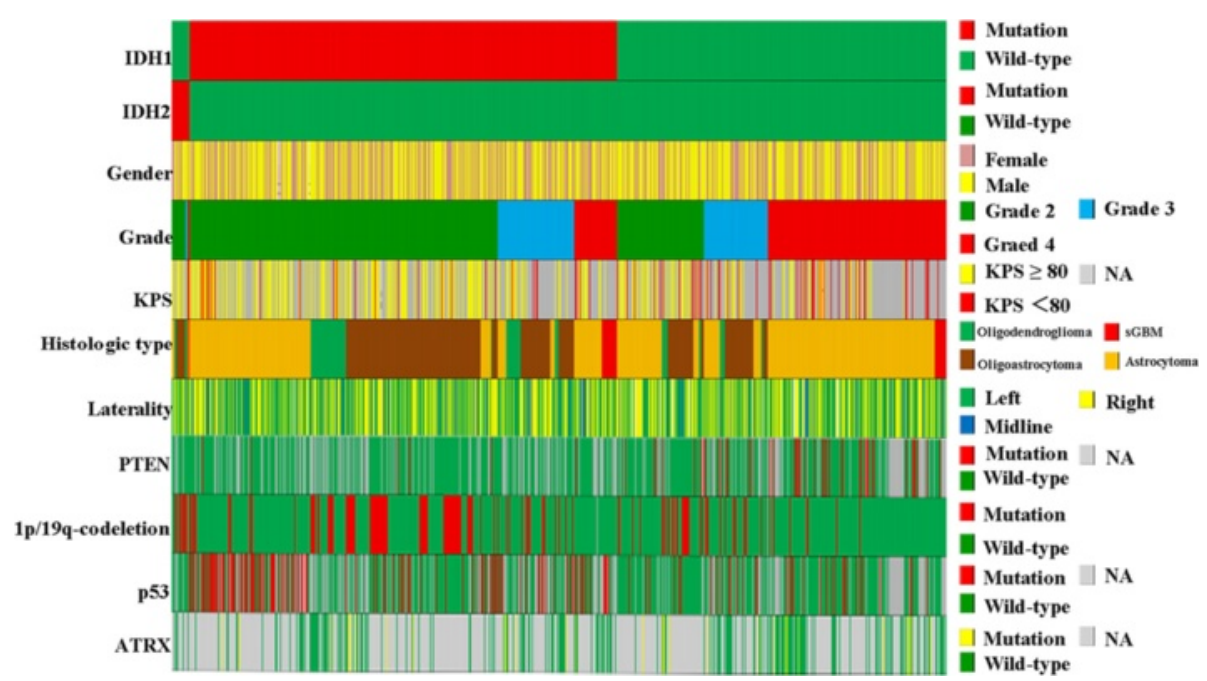

Fig. 1 Overview of clinical and molecular characterization of the cohort $(n=811)$. Each column represents a patient

Clinicopathologic data, including gender, age, pathologic diagnosis and the results of molecular analysis were obtained. When the cases were classified as secondary GBMs based on biopsy-proven preexisting low-grade gliomas, 29 cases $(12.4 \%)$ were secondary GBM and the remainder were primary GBM (205 cases, $87.6 \%$ ).

Whole transcriptome sequencing of 161 gliomas and DNA methylation profile of 44 glioma samples, were obtained from Chinese Glioma Genome Atlas (CGGA) database (http://www.cgga.org.cn) [11-13]. All these samples were histologically graded according to 2007 WHO classification of tumours of the nervous systems [6]. Written informed consent was obtained from all donors. Clinical investigations were performed after approval by the local research ethics committee and in accordance with the ethical principles.

\section{IDH mutation}

Genomic DNA was isolated from frozen tissues with a QIAamp DNA Mini Kit (Qiagen) according to the manufacturer's protocol. The DNA concentration and quality were evaluated with a Nano-Drop ND-1000 spectrophotometer (NanoDrop Technologies, Houston, TX). The pyrosequencing of $I D H 1 / 2$ mutations was supported by Gene-tech (Shanghai, China) and performed on a PyroMark Q96 ID System (Qiagen, Valencia, Calif). The primers 5' -GCTTGTGAGTGGATGGGTAAAAC-3', 5' '-Biotin-TT GCCAACATGACTTACTTGATC-3', for $I D H 1$ and $5^{\prime}$-AT CCTGGGGGGGACTGTCTT-3', 5'-Biotin-CTCTCCAC CCTGGCCTACCT-3' for IDH2 were used for PCR amplification, and the primers 5'-TGGATGGGTAAAACCT-3' for IDH1 and 5'-AGCCCATCACCATTG-3' for IDH2 were used for pyrosequencing [13].

\section{Gene set enrichment analysis}

To identify the gene sets related to particular biological processes present in IDH-mutant patients, gene expression profiling and a gene set enrichment analysis (GSEA) were performed as described previously [14].

\section{Statistical analysis}

Survival distributions were estimated with a Kaplan-Meier survival analysis, and the log-rank test was used to assess the significance of differences between stratified survival groups using the GraphPad Prism 5.0 statistical software. The differences among patients in baseline clinical and molecular features according to IDH1 and $I D H 2$ mutational status were tested using the Fisher's exact and Wilcoxon rank sum tests for categoric and continuous variables, respectively. Gens that were differently methylated between IDH2 mutant and IDH1 mutant tumors were obtained using the standard two-sampled $t$-test with unequal variance and sample size. To adjust for multiple comparisons, we applied the Benjamini-Hochberg method to control the False Discovery Rate at $5 \%$. We further filtered the list of significant genes by retaining those which exhibited at least 1.5-fold difference in gene expression between IDH2 mutant and IDH1 mutant in our final comparisons. Student's $t$-test was performed using SPSS 16.0. A two-sided $p$ value $<0.05$ was considered significant.

\section{Results}

Clinical and molecular characterization of $I D H 2$ mutations Among a total of 811 gliomas, IDH2 mutations were identified in 18 cases (2.2\%) (Table 1). IDH2 mutations were found in $0.5 \%$ of pGBM (1/215), $3.4 \%$ of sGBM $(1 / 29)$ and $2.8 \%(16 / 577)$ of low grade gliomas, while 
Table 2 Clinical characteristics according to IDH mutational status in gliomas

\begin{tabular}{|c|c|c|c|c|c|c|c|c|}
\hline \multirow[t]{2}{*}{ Clinical characteristic } & & \multirow[t]{2}{*}{$N=811$} & \multirow{2}{*}{$\begin{array}{l}\text { IDH1-Mutated } \\
\text { No. (\%) }\end{array}$} & \multirow{2}{*}{$\begin{array}{l}\text { IDH2-Mutated } \\
\text { No. (\%) }\end{array}$} & \multirow{2}{*}{$\begin{array}{l}\text { IDH1/IDH2-Wild-type } \\
\text { No. (\%) }\end{array}$} & \multirow[t]{2}{*}{$\begin{array}{l}\text { P (IDH1-Mutated VS } \\
\text { IDH1/IDH2- Wild-type) }\end{array}$} & \multirow[t]{2}{*}{$\begin{array}{l}\text { P (IDH2-Mutated VS IDH1/ } \\
\text { IDH2-Wild-type) }\end{array}$} & \multirow[t]{2}{*}{$\begin{array}{l}\text { P (IDH1-Mutated VS } \\
\text { IDH2-Mutated) }\end{array}$} \\
\hline & & & & & & & & \\
\hline \multirow[t]{2}{*}{ Age, years } & $<45$ & 423 & $285(67.4)$ & $13(3.1)$ & $125(29.5)$ & \multirow[t]{2}{*}{$<0.001$} & \multirow[t]{2}{*}{$<0.05$} & \multirow[t]{2}{*}{0.456} \\
\hline & $\geq 45$ & 388 & $163(42.0)$ & $5(1.3)$ & $220(56.7)$ & & & \\
\hline \multicolumn{3}{|l|}{ Median age, years } & 39.1 & 37.3 & 45.0 & \multirow[t]{2}{*}{$<0.001$} & \multirow[t]{2}{*}{$<0.05$} & \multirow[t]{2}{*}{0.456} \\
\hline Range & & & $17.0-66.0$ & $26.0-56.0$ & $9.0-81.0$ & & & \\
\hline \multirow[t]{2}{*}{ Gender } & Male & 495 & $265(53.5)$ & $11(2.2)$ & $219(44.2)$ & \multirow[t]{2}{*}{0.113} & \multirow[t]{2}{*}{0.801} & \multirow[t]{2}{*}{0.824} \\
\hline & Female & 316 & $186(58.9)$ & $7(2.2)$ & $123(38.9)$ & & & \\
\hline \multirow[t]{2}{*}{ WHO Grade } & $\|+\|$ & 577 & $403(69.8)$ & $16(2.8)$ & $158(27.4)$ & \multirow[t]{2}{*}{$<0.001$} & \multirow[t]{2}{*}{$<0.001$} & \multirow[t]{2}{*}{0.883} \\
\hline & IV & 234 & $45(19.2)$ & $2(0.9)$ & $187(79.9)$ & & & \\
\hline \multirow[t]{3}{*}{ KPS score } & $<80$ & 74 & $26(35.1)$ & $2(2.7)$ & $46(62.2)$ & \multirow[t]{3}{*}{$<0.001$} & \multirow[t]{3}{*}{0.376} & \multirow[t]{3}{*}{0.514} \\
\hline & $\geq 80$ & 253 & $173(68.4)$ & $7(2.8)$ & $73(28.8)$ & & & \\
\hline & NA & 483 & 248 & 9 & 226 & & & \\
\hline \multirow[t]{4}{*}{ Histologic type } & Oligodendroglioma & 76 & $57(75.0)$ & $4(5.3)$ & $15(19.7)$ & \multirow[t]{4}{*}{$<0.001$} & \multirow[t]{4}{*}{$<0.001$} & \multirow[t]{4}{*}{0.218} \\
\hline & Oligoastrocytoma & 269 & $194(72.1)$ & $10(3.7)$ & $65(24.2)$ & & & \\
\hline & Astrocytoma & 437 & $181(41.4)$ & $3(0.7)$ & $253(57.9)$ & & & \\
\hline & sGBM & 29 & $16(55.2)$ & $1(3.4)$ & $12(41.4)$ & & & \\
\hline \multirow[t]{4}{*}{ Laterality } & Left & 380 & 211 (55.5) & $9(2.4)$ & $160(42.1)$ & \multirow[t]{4}{*}{$<0.05$} & 0.891 & 0.985 \\
\hline & Right & 366 & $203(55.5)$ & $8(2.2)$ & $155(42.3)$ & & & \\
\hline & Midline & 53 & $33(62.3)$ & $1(1.9)$ & 19 (35.8) & & & \\
\hline & NA & 12 & 1 & 0 & 11 & & & \\
\hline
\end{tabular}


Table 3 Molecular characteristics according to IDH mutation in glioma

\begin{tabular}{|c|c|c|c|c|c|c|c|}
\hline Molecular Characteristic & & IDH1-Mutated & IDH2-Mutated & IDH1/IDH2-WT & $\begin{array}{l}\text { P (IDH1-Mutated } \\
\text { VS IDH1/IDH2-WT) }\end{array}$ & $\begin{array}{l}\text { P (IDH2-Mutated } \\
\text { VS IDH1/IDH2-WT) }\end{array}$ & $\begin{array}{l}\text { P (IDH1-Mutated } \\
\text { VS IDH2-Mutated) }\end{array}$ \\
\hline \multirow[t]{3}{*}{ PTEN } & Mutation & 6 & 0 & 43 & $<0.001$ & 0.098 & 0.605 \\
\hline & Wild-type & 314 & 14 & 218 & & & \\
\hline & NA & 128 & 4 & 84 & & & \\
\hline \multirow[t]{3}{*}{ 1p/19q Co-deletion } & Absent & 341 & 9 & 307 & $<0.001$ & $<0.001$ & $<0.05$ \\
\hline & Present & 106 & 9 & 34 & & & \\
\hline & NA & 1 & 0 & 1 & & & \\
\hline \multirow[t]{3}{*}{ P53 } & Mutation & 215 & 0 & 232 & $<0.001$ & 0.154 & $<0.05$ \\
\hline & Wild-type & 115 & 14 & 34 & & & \\
\hline & NA & 118 & 4 & 79 & & & \\
\hline \multirow[t]{3}{*}{ ATRX } & Mutation & 13 & 0 & 15 & 0.084 & 0.638 & 0.728 \\
\hline & Wild-type & 107 & 5 & 102 & & & \\
\hline & NA & 328 & 13 & 228 & & & \\
\hline
\end{tabular}

IDH1 mutations are found in $14.1 \%(29 / 205)$ of pGBM, $55.2 \%(16 / 29)$ of sGBM and $69.8 \%(403 / 577)$ of low grade gliomas. Combined $I D H 1$ and $I D H 2$ mutations were found in $14.6 \%(30 / 205)$ of pGBM, $58.6 \%(17 / 29)$ of sGBM and $72.6 \%(419 / 577)$ of low grade gliomas.

As shown in Fig. 1 and Table 2, patients with mutations in $I D H 2$ did not differ from IDH1-mutant patients in terms of age, gender, WHO grade, KPS, histologic type and laterality (Table 2). To characterize the molecular features of $I D H 2$ mutant gliomas, we analyzed associations between IDH2 mutations and other mutational events. Patients with IDH2 mutations had a higher frequency of $1 p / 19 q$ co-deletion $(p<0.05)$ and a lower frequency of P53 mutation $(p<0.05)$ than IDH1 mutant patients (Table 3 ). Strikingly, the presence of IDH2 mutations and PTEN mutations, P53 mutation and ATRX mutation did not correlate (Fig. 1 and Table 3).

\section{Gene set enrichment analysis for IDH2 mutant patients}

To gain biologic insight into the potentially significance of IDH2 mutations, we compared the whole-transcriptome sequencing expression profiles of $5 \mathrm{IDH} 2$ mutant patients with $109 \mathrm{IDH} 1$ mutant patients and $47 \mathrm{IDH} 1 / 2$ wild-type patients. First, we used a Gene Set Enrichment Analysis (GSEA) to compare the global gene expression profiles of the IDH2 mutant and IDH1 mutant gliomas. The result showed that the oxidative phosphorylation gene set was upregulated (FDR q-value $=0$; Fig. $2 \mathrm{a} / \mathrm{c}$ ). We then compared the whole-transcriptome sequencing expression profiles of the $I D H 2$ mutant and $I D H 1 / 2$ wild-type gliomas (Fig. 2b). The results showed that the oxidative phosphorylation gene set (FDR q-value $<0.001$; Fig. $2 \mathrm{~d}$ ) and hedgehog signaling set were upregulated (FDR q-value $<0.05$; Fig. 2e).
IDH2 mutant gliomas exhibit DNA methylation profiles distinct from those of $\mathrm{IDH} 1$ mutant gliomas

Given the DNA methylation profiles of $3 \mathrm{IDH} 2$ mutant gliomas and 41 IDH1 mutant gliomas, we used standard t-tests to identify differentially methylated regions. The methylation patterns of genes that correlated with IDH 2 mutant gliomas are shown in Fig. 3a using a onedimensional hierarchical clustering analysis. The four most representative biological processes for genes commonly altered by hypermethylation were the regulation of cell proliferation, cell motion, cell migration and response to hypoxia (Fig. 3b). According to the hypomethylated genes, the three most representative biological processes were ion transport, cell-cell signaling, and cation transport (Fig. 3b).

\section{Associations of IDH2 mutations with clinical outcome}

In our cohort, the presence of an IDH2 mutation was associated with a longer overall survival $(p<0.05)$ and longer progression-free survival $(p<0.05)$ (Fig. $4 \mathrm{a} / \mathrm{b})$ than the presence of the IDH1/2 wild-type gene. However, when considering all patients with IDH2 mutations, the overall survival and time to recurrence did not differ from those of IDH1 mutant patients (Fig. 4a/b). This result illustrates that the effects of IDH 2 mutation and IDH1 mutation on clinical prognosis were similar.

\section{Discussion}

Mutations in the IDH1 and IDH2 genes have been found in patients with gliomas and were initially identified in low-grade gliomas and secondary glioblastomas [1]. Strikingly, mutations in $I D H 1$ and $I D H 2$ are mutually exclusive in gliomas. Although the genetic and epigenetic landscapes of IDH1 mutation gliomas have been extensively 


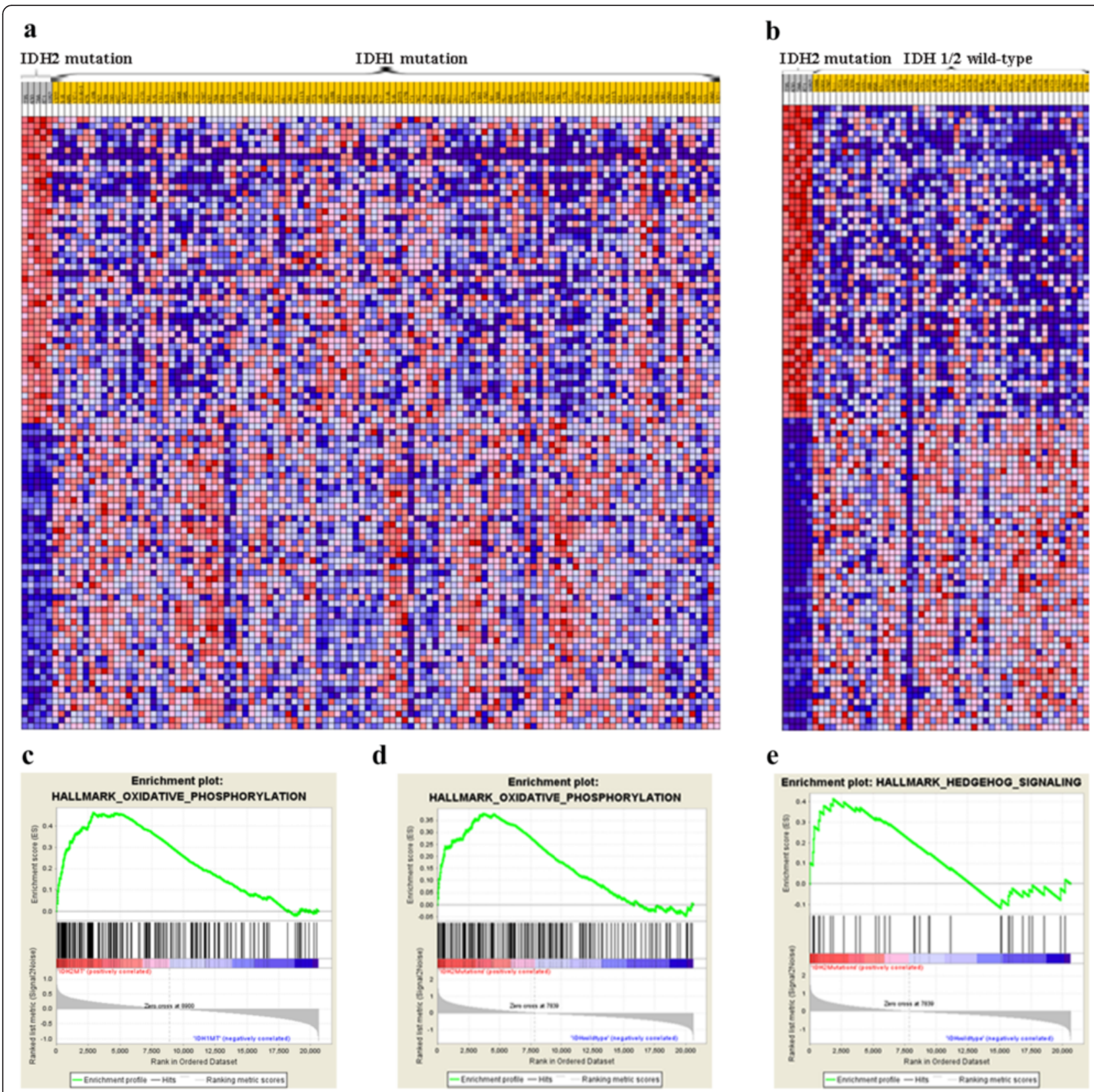

Fig. 2 Gene Set Enrichment Analysis (GSEA) of overexpressed genes in glioma harboring IDH2 mutations. Each row represents a gene, and each column indicates a glioma with an IDH2 mutation, IDH1 mutation or IDH1/2 wild-type. Red indicates upregulated genes, and blue indicates downregulated genes. a Expression levels of genes annotated in IDH2 mutant gliomas compared to IDH1 mutant gliomas. b Expression levels of genes annotated in IDH2 mutant gliomas compared to IDH1/2 wild-type gliomas. c one representative plot of GSEA from a. d-e two representative plots of GSEA from (b)

studied, whether IDH2 mutation gliomas have unique genetic and epigenetic characteristics that can be used as targets for future intervention is unknown. In this report, we compared the clinical and molecular characteristics of glioma patients harboring $I D H 1$ and $I D H 2$ mutations.

Like mutations in $I D H 1$, mutations in $I D H 2$ affect a conserved arginine residue (R172) in the substrate-binding site of the IDH2 enzyme. In our cohort, the presence of an
IDH2 mutation did not correlate with the presence of PTEN, P53, and ATRX mutations, but a highly significant positive correlation was observed with the presence of a $1 p / 19 q$ co-deletion: $44.4 \%$ of $I D H 2$ mutation patients harbored a $1 p / 19 q$ co-deletion. In malignant glioma, $I D H 1$ mutations are ubiquitous in tumor cells, and IDH1 mutations precede secondary and tertiary lesions, suggesting that IDH1 mutations are an early causative event in the 


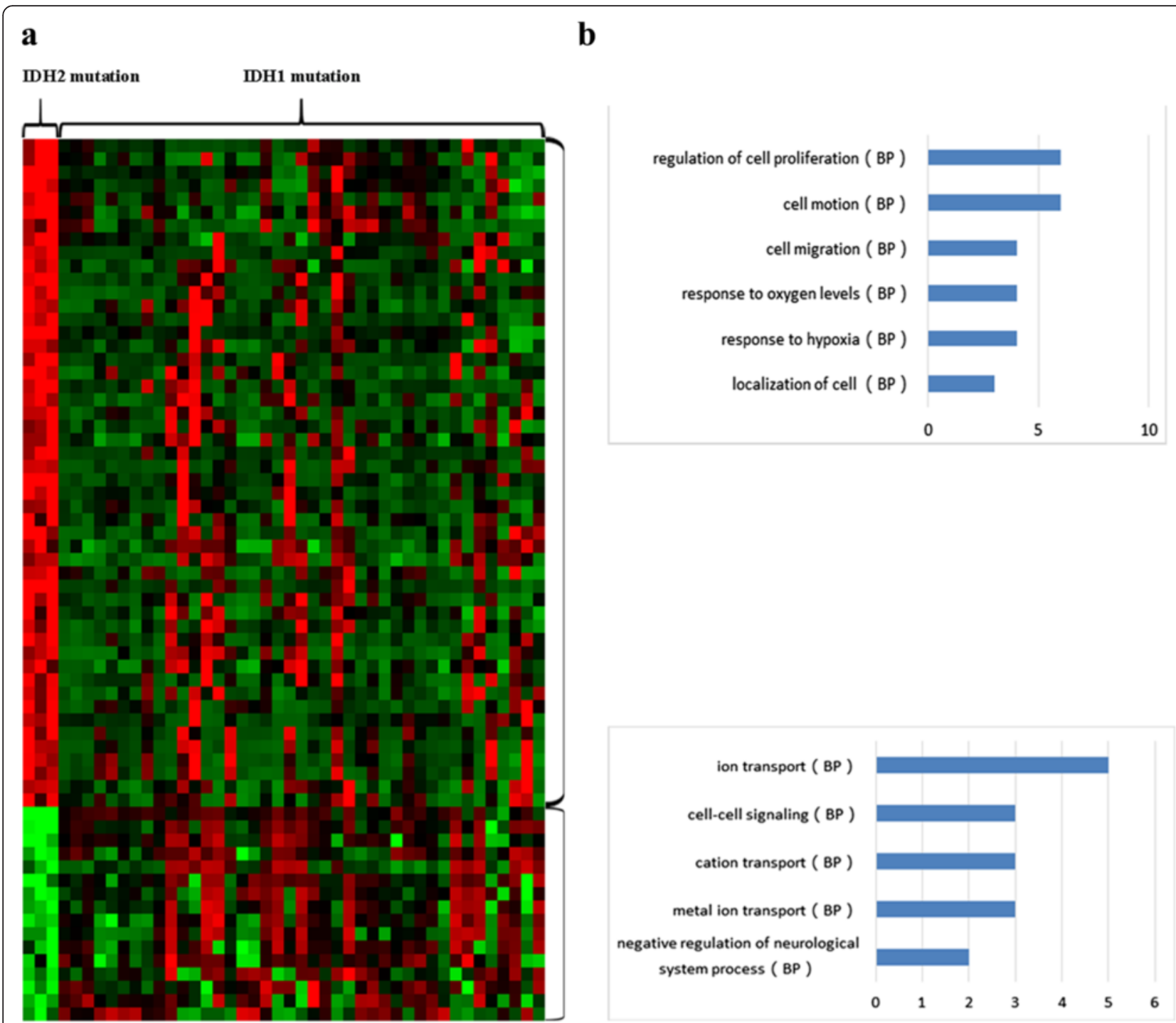

Fig. 3 Clustering analysis of DNA methylation in IDH2 mutant gliomas. a The pattern of DNA methylation was associated with IDH2 mutant gliomas using a one-dimensional hierarchical clustering analysis. b Functional enrichment analysis of associated genes, indicating the functional roles of gene sets in different subgroups. Enrichment results for biological processes were obtained from the GO database. The orders of biological processes listed in the histogram are based on the number of targets annotated in the biological process (BP)
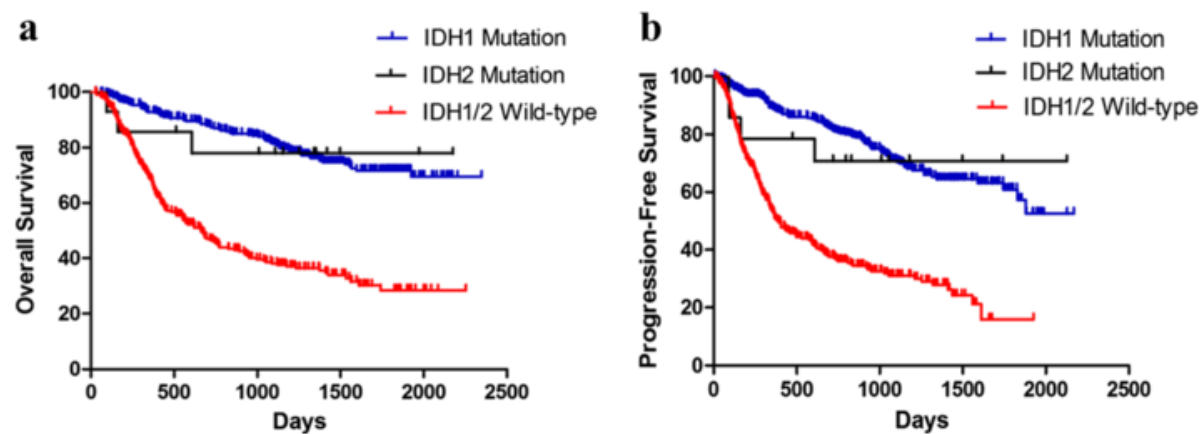

Fig. 4 The Kaplan-Meier estimates for Overall survival (OS) (a) and Progression-free survival (PFS) (b) indicates that IDH2 mutant gliomas associated with longer overall survival $(p=0.011)$ and longer progression-free survival $(p=0.011)$ than IDH1/2 wild-type gliomas. However, the OS and PFS did not significantly differ between IDHI mutant and IDH2 mutant gliomas 
genesis of gliomas [15-17]. A pathology study of multiple biopsies from the same patient found that IDH1 mutations occurred before the acquisition of P53 mutations and $1 p / 19 q$ loss of heterozygosity (LOH) [16], suggesting that IDH1 mutations may result in cellular stress that leads to the mutation of $P 53$ and $1 p / 19 q$ loss. However, IDH 2 mutations and PTEN, P53 and ATRX mutations were mutually exclusive, suggesting that the microenvironment of IDH2 mutations may not create cellular stress that leads to the other mutations, which needs further research to fully elucidate.

Tumor cells often take up nutrients in excess of their bioenergetic needs and shunt metabolites into pathways that support tumor progression [18-20]. During cell proliferation, tumor cells depend on aerobic glycolysis to meet their bioenergy needs and generate intermediates for macromolecule biosynthesis. One study demonstrated that glioma cells harboring mutant IDH1 may maintain cell proliferation via the glutamate metabolism pathway [21]. In our study, GSEA was performed for $I D H 2$ and IDH1 mutations, yielding enriched gene sets related to oxidative phosphorylation, which is critical to tricarboxylic acid (TCA) cycle, in the IDH2 mutation subset. This finding corroborates that of a previous study [22, 23]. IDH2 is localized in the mitochondria and participates in the TCA to produce energy, whereas $I D H 1$ is localized in the cytoplasm and peroxisomes [24]. Consequently, does energy production in IDH2-mutated gliomas favor oxidative phosphorylation over aerobic glycolysis? These interesting findings should be verified in more cases before accepting them as general characteristics of IDH2-mutated gliomas. Future work should focus on the potential of therapeutically targeting compensatory metabolic pathways in $\mathrm{IDH} 2$ mutant gliomas.

\section{Conclusion}

In conclusion, our results describe the clinical and biological characteristics of $I D H 1$ and $I D H 2$ mutations in gliomas. Understanding the underlying biology of the differences in outcome observed for IDH1 and IDH2 mutant gliomas will be important for future studies and may lead to the development of novel approaches to therapy.

\footnotetext{
Abbreviations

CGGA: Chinese Glioma Genome Atlas; GSEA: gene set enrichment analysis; $\mathrm{IDH} 1$ : isocitrate dehydrogenase $1 ; \mathrm{IDH} 2$ : isocitrate dehydrogenase 2; OS: overall survival; PFS: progression-free survival; sGBM: secondary glioblastomas.
}

\section{Acknowledgments}

This study was funded by the following grants: 1. Guangdong Provincial Clinical Medical Centre for Neurosurgery (No. 2013B020400005). 2. Beijing science and technology plan (No. Z131100006113018). 3. National Natural Science Foundation of China (No. 81371397, 91229121, 81272804, 81071011). 4. National High Technology Research and Development Program (No. 2012AA02A508), International Science and Technology Cooperation Program (No.2012DFA30470). 5. National High Technology Research and Development Program (No. 2012AA02A508).

\section{Authors' contributions}

HYW, KT, TYL conceived of the study and draft the manuscript. WZZ, JYL, WW, HMH helped to collect the clinical data of patients. MYL, HQW, XZH ZYZ helped to performed the statistical analysis. SZZ and YWL participated in its design. All authors read and approved the final manuscript.

\section{Competing interests}

The authors declare that they have no competing interests.

\section{Author details}

'Department of Neurosurgery, Zhujiang Hospital, Southern Medical University, 253\# Gongye Road, Guangzhou, China. ${ }^{2}$ Department of Neurosurgery, Beijing Tiantan Hospital, Capital Medical University, No. 6 TiantanXili, Dongcheng District, Beijing 100050, China. ${ }^{3}$ The National Key Clinical Specialty. The Engineering Technology Research Center of Education Ministry of China Guangdong Provincial Key Laboratory on Brain Function Repair and Regeneration Department of Neurosurgery, Zhujiang Hospital, Southern Medical University, Guangzhou, China. ${ }^{4}$ Beijing Neurosurgical Institute, Capital Medical University, Beijing, China. ${ }^{5}$ Chinese Glioma Cooperative Group (CGCG), Beijing, China. ${ }^{6}$ Center for Brain Disorders Research, Capital Medical University, Beijing 100069, China.

Received: 24 February 2016 Accepted: 17 May 2016

Published online: 31 May 2016

\section{References}

1. De Carli E, Wang X, Puget S. IDH1 and IDH2 mutations in gliomas. N Engl J Med. 2009;360:2248. author reply 2249.

2. Wang $P$, Dong Q, Zhang C, Kuan PF, Liu Y, Jeck WR, et al. Mutations in isocitrate dehydrogenase 1 and 2 occur frequently in intrahepatic cholangiocarcinomas and share hypermethylation targets with glioblastomas. Oncogene. 2013;32:3091-100

3. Marcucci G, Maharry K, Wu YZ, Radmacher MD, Mrozek K, Margeson D, et al. IDH1 and IDH2 gene mutations identify novel molecular subsets within de novo cytogenetically normal acute myeloid leukemia: a Cancer and Leukemia Group B study. J Clin Oncol. 2010;28:2348-55.

4. Amary MF, Bacsi K, Maggiani F, Damato S, Halai D, Berisha F, et al. IDH1 and IDH2 mutations are frequent events in central chondrosarcoma and central and periosteal chondromas but not in other mesenchymal tumours. J Pathol. 2011;224:334-43.

5. Shibata T, Kokubu A, Miyamoto M, Sasajima Y, Yamazaki N. Mutant IDHI confers an in vivo growth in a melanoma cell line with BRAF mutation. Am J Pathol. 2011;178:1395-402.

6. Louis DN, Ohgaki H, Wiestler OD, Cavenee WK, Burger PC, Jouvet A, et al. The 2007 WHO classification of tumours of the central nervous system. Acta Neuropathol. 2007;114:97-109.

7. Weller M, Cloughesy T, Perry JR, Wick W. Standards of care for treatment of recurrent glioblastoma-are we there yet. Neuro Oncol. 2013;15:4-27.

8. Parsons DW, Jones S, Zhang X, Lin JC, Leary RJ, Angenendt P, et al. An integrated genomic analysis of human glioblastoma multiforme. Science. 2008:321:1807-12.

9. Shen Y, Zhu YM, Fan X, Shi JY, Wang QR, Yan XJ, et al. Gene mutation patterns and their prognostic impact in a cohort of 1185 patients with acute myeloid leukemia. Blood. 2011;118:5593-603.

10. Ward PS, Lu C, Cross JR, Abdel-Wahab O, Levine RL, Schwartz GK, et al. The potential for isocitrate dehydrogenase mutations to produce 2hydroxyglutarate depends on allele specificity and subcellular compartmentalization. J Biol Chem. 2013;288:3804-15.

11. Bao ZS, Chen HM, Yang MY, Zhang CB, Yu K, Ye WL, et al. RNA-seq of 272 gliomas revealed a novel, recurrent PTPRZ1-MET fusion transcript in secondary glioblastomas. Genome Res. 2014;24(11):1765-73.

12. Zhang W, Yan W, You G, Bao Z, Wang Y, Liu Y, et al. Genome-wide DNA methylation profiling identifies ALDH1A3 promoter methylation as a prognostic predictor in G-CIMP- primary glioblastoma. Cancer Lett. 2013; 328:120-5.

13. Cai J, Chen J, Zhang W, Yang P, Zhang C, Li M, et al. Loss of ATRX, associated with DNA methylation pattern of chromosome end, impacted biological behaviors of astrocytic tumors. Oncotarget. 2015;6:18105-15.

14. Subramanian A, Tamayo P, Mootha VK, Mukherjee S, Ebert BL, Gillette MA, et al. Gene set enrichment analysis: a knowledge-based approach for 
interpreting genome-wide expression profiles. Proc Natl Acad Sci U S A. 2005;102:15545-50.

15. Lai A, Kharbanda S, Pope WB, Tran A, Solis OE, Peale F, et al. Evidence for sequenced molecular evolution of IDH1 mutant glioblastoma from a distinct cell of origin. J Clin Oncol. 2011;29:4482-90.

16. Watanabe T, Nobusawa S, Kleihues P, Ohgaki H. IDH1 mutations are early events in the development of astrocytomas and oligodendrogliomas. Am J Pathol. 2009;174:1149-53.

17. Wakimoto H, Tanaka S, Curry WT, Loebel F, Zhao D, Tateishi K, et al. Targetable signaling pathway mutations are associated with malignant phenotype in IDH-mutant gliomas. Clin Cancer Res. 2014;20:2898-909.

18. Benjamin DI, Cravatt BF, Nomura DK. Global profiling strategies for mapping dysregulated metabolic pathways in cancer. Cell Metab. 2012;16:565-77.

19. Qin LS, Jia PF, Zhang ZQ, Zhang SM. ROS-p53-cyclophilin-D signaling mediates salinomycin-induced glioma cell necrosis. J Exp Clin Cancer Res. 2015;34:57.

20. Cheng Z, Wang HZ, Li X, Wu Z, Han Y, Li Y, et al. MicroRNA-184 inhibits cell proliferation and invasion, and specifically targets TNFAIP2 in Glioma. J Exp Clin Cancer Res. 2015;34:27.

21. Seltzer MJ, Bennett BD, Joshi AD, Gao P, Thomas AG, Ferraris DV, et al. Inhibition of glutaminase preferentially slows growth of glioma cells with mutant IDH1. Cancer Res. 2010:70:8981-7.

22. Waitkus MS, Diplas BH, Yan H. Isocitrate dehydrogenase mutations in gliomas. Neuro Oncol. 2016;18(1):16-26.

23. Reitman ZJ, Jin G, Karoly ED, Spasojevic I, Yang J, Kinzler KW, et al. Profiling the effects of isocitrate dehydrogenase 1 and 2 mutations on the cellular metabolome. Proc Natl Acad Sci U S A. 2011;108:3270-5.

24. Xu X, Zhao J, Xu Z, Peng B, Huang Q, Arnold E, et al. Structures of human cytosolic NADP-dependent isocitrate dehydrogenase reveal a novel selfregulatory mechanism of activity. J Biol Chem. 2004;279:33946-57.

\section{Submit your next manuscript to BioMed Central} and we will help you at every step:

- We accept pre-submission inquiries

- Our selector tool helps you to find the most relevant journal

- We provide round the clock customer support

- Convenient online submission

- Thorough peer review

- Inclusion in PubMed and all major indexing services

- Maximum visibility for your research

Submit your manuscript at www.biomedcentral.com/submit

) Biomed Central 